\title{
General machine learning model, review, and experimental- theoretic study of magnolol activity in enterotoxigenic induced oxidative stress
}

\author{
Yanli Deng ${ }^{1,4}$, Yong Liu ${ }^{2,5}$, Shaoxun Tang ${ }^{2,3}$, Chuanshe Zhou ${ }^{2,3}$, Xuefeng Han ${ }^{2}$, \\ Wenjun Xiao ${ }^{1}$, Lucas Anton Pastur-Romay ${ }^{5,6}$, José Manuel Vázquez-Naya ${ }^{5,6}$, \\ Javier Pereira Loureiro $^{7}$, Cristian R. Munteanu ${ }^{5}$ and Zhiliang Tan ${ }^{2,3}$
} ${ }^{1}$ National Research Center of Engineering Technology for Utilization of Botanical Functional Ingredients,
Hunan Agricultural University, Changsha, Hunan 410128, P.R. China; ${ }^{2}$ Key Laboratory for Agro-
Ecological Processes in Subtropical Region, Hunan Research Center of Livestock and Poultry Sciences,
South Central Experimental Station of Animal Nutrition and Feed Science in the Ministry of Agriculture,
Institute of Subtropical Agriculture, The Chinese Academy of Sciences, Changsha, Hunan 410125, P.R.
China; ${ }^{3}$ Hunan Co-Innovation Center of Animal Production Safety, CICAPS, Changsha, Hunan 410125,
P.R. China; ${ }^{4}$ Tea College of Guizhou University, Guizhou, 550025, P.R. China; ${ }^{2}$ RNASA-IMEDIR,
Computer Science Faculty, University of A Coruna, Campus de Elviña s/n, 15071, A Coruña, Spain; ${ }^{6}$
Instituto de Investigación Biomédica de A Coruña (INIBIC), Complexo Hospitalario Universitario de A
Coruña (CHUAC), A Coruña, 15006, Spain; ${ }^{7}$ RNASA-IMEDIR, Faculty of Health Science, University of A
Coruña, Coruña, Spain

\begin{abstract}
This study evaluated the antioxidative effects of magnolol based on the mouse model induced by Enterotoxigenic Escherichia coli (E. coli, ETEC). All experimental mice were equally treated with ETEC suspensions $(3.45 \times 109 \mathrm{CFU} / \mathrm{ml})$ after oral administration of magnolol for 7 days at the dose of $0,100,300$ and $500 \mathrm{mg} / \mathrm{kg}$ Body Weight (BW), respectively. The oxidative metabolites and antioxidases for each sample (organism of mouse) were determined: Malondialdehyde (MDA), Nitric Oxide (NO), Glutathione (GSH), Myeloperoxidase (MPO), Catalase (CAT), Superoxide Dismutase (SOD), and Glutathione Peroxidase (GPx). In addition, we also determined the corresponding mRNA expressions of CAT, SOD and GPx as well as the Total Antioxidant Capacity (T-AOC). The experiment was completed with a theoretical study that predicts a series of 79 ChEMBL activities of magnolol with 47 proteins in 18 organisms using a Quantitative StructureActivity Relationship (QSAR) classifier based on the Moving Averages (MAs) of Rcpi descriptors in three types of experimental conditions (biological activity with specific units, protein target and organisms). Six Machine Learning methods from Weka software were tested and the best QSAR classification model was provided by Random Forest with True Positive Rate (TPR) of 0.701 and Area under Receiver Operating Characteristic (AUROC) of 0.790 (test subset, 10-fold crossvalidation). The model is predicting if the new ChEMBL activities are greater or lower than the average values for the magnolol targets in different organisms.
\end{abstract}

Keywords: QSAR model, Magnolol, Antioxidative activity, Reactive oxygen species, Machine learning, Random forest. 


\section{INTRODUCTION}

Biological systems are frequently exposed to excessive reactive oxygen species (ROS), causing a disturbance into the natural antioxidant defense systems and, therefore, resulting in damage to all biomolecules, including nucleic acids, proteins, and lipids [1]. It is well known that lipopolysaccharides are capable of causing a wide variety of pathophysiological effects, due to the excessive production of proinflammatory cytokines and mediators, and the increased production of superoxide radical, lipid peroxides and nitric oxide results in ROS products [2]. Any additional burden of free radicals in animal and human physiological systems can tip the pro-oxidant and antioxidant balance, leading to oxidative stress. Currently, the antioxidant enzymes and lowmolecular- weight antioxidants are the two major antioxidant defense systems in animals and humans [3,4]. Specifically, the compound magnolol is a main bioactive ingredient isolated from the cortex of Magnolol officinalis [5]. Accumulating evidence indicates that magnolol may play an important role in the treatment of various diseases due to its anticancer [6], anti-clastogenic [7], anti-inflammatory effects [8], and antioxidant activity, etc. [9-18]. It is noteworthy that the relevant magnolol studies have mainly focused on the evaluation of its beneficial effects against oxidative radicals in vitro. However, few studies have been conducted to comprehensively investigate the effects of magnolol on the antioxidant enzyme system in relation to oxidative stress in vivo.

Consequently, the objectives of this study were the following. First, we carried out a short review about the activity of magnolol. Next, we reported new experimental studies to determine the anti-oxidative effects of magnolol on oxidative status, antioxidant enzyme activities, and antioxidant enzyme mRNA and protein expression in the livers of Enterotoxigenic Escherichia coli (ETEC)-induced and non-ETEC induced mice. Last, the experimental results are extended with a computational study for the prediction of magnolol biological activities against the protein targets in different organisms. Thus, a Quantitative Structure-Activity Relationships (QSAR) [19, 20] was obtained as a Machine Learning [21] classifier that is able to predict that a specific ChEMBL activity [22] against a specific protein target into an organism is greater or lower than the average value for this activity in the dataset. Therefore, these predictions are completing the current experimental results in rat with new biological activities in new organisms, without the necessity of all the experimental combinations. This way, the theoretical method is a fast and cheap alternative to the experiments.

The QSAR model is using as features the molecular descriptors [23] of the compounds that interacts with the protein targets. The coding of the molecular information into molecular descriptors has been demonstrated as a powerful method to create QSAR models for drugs, proteins or nucleic acids [24-26]: antiviral drugs [27-29], anti-microbial compounds [30-35], proteins [36-41], nucleic acids [42, 43]. The current study used compound descriptors and their moving averages (MAs) in three experimental conditions such as measuring ChEMBL activity (with units), protein target, and organism.

\section{SHORT REVIEW OF MAGNOLOL ACTIVITY}

Magnolol is the main bioactive ingredient isolated from the cortex of Magnolia officinalis [5]. Magnolol has been proved to act a vital role in dealing with various diseases. As a low-molecularweight antioxidant, magnolol is a hydroxylated biphenyl that can donate hydrogen atoms to the damaged molecules. Several previous studies have demonstrated that magnolol has a strong antioxidant ability to suppress the oxidation of lipid peroxidation [9] or to increase SOD activity to attenuate the oxidative damage [10]. ROS are an important inducer of the pathogenesis of these diseases and the therapeutic function of magnolol is tightly linked to its antioxidant effectiveness. Moreover, it has been reported that magnolol scavenges hydroxyl radical [11], peroxy-nitrite [12] and hydrogen peroxide [13] to reduce or suppress the generation of ROS. Furthermore, there is an 
additional direct evidence of its anti-oxidative effect on intracellular glutathione depletion [9] or enzymatic system capacity in mice [14].

Lo et al. [15] demonstrated that magnolol and honokiol from Magnolia officinalis can protect rat heart mitochondria against lipid peroxidation. Thus, lipid peroxidation was induced in isolated rat heart mitochondria using Adenosine Diphosphate (ADP) and Ferrous Sulfate (FeSO4). The study showed that the antioxidant effect of magnolol was 1,000 times higher than that of $\alpha$ tocopherol and the magnolol demonstrated more free radical scavenging activity but less potent than $\alpha$-tocopherol.

Ke Yao et al. [16] used the Human Lens Epithelial (HLE) cells to study the protective effect of magnolol against hydrogen peroxide-induced oxidative stress. This study was determined by the role of the oxidative stress in the cataract's progression. Thus, magnolol presented a protection effect $\mathrm{H}_{2} \mathrm{O}_{2}$-induced cytotoxicity in this type of cells by the inhibition of the ROS, the loss of mitochondrial membrane potential and the release of cytochrome c from mitochondria. Other effects of magnolol were observed: the inhibition of the $\mathrm{H}_{2} \mathrm{O}_{2}$-induced expressions of caspase-9 and caspase-3, the attenuation of Extracellular Signal-Regulated Kinase/ Mitogen Activated Protein Kinase (ERK/MAPK) deactivation. In conclusion, magnolol was proposed as a protector against cataractogenesis.

Another magnolol study demonstrated its neuroprotective activity in cultured rat cerebellar granule cell damage [17]. The neuron toxicity was induced by glucose deprivation, excitatory amino acids and hydrogen peroxide. As conclusion, the study showed that magnolol alone did not affect mitochondrial function or cell damage. Opposite, magnolol significantly reversed glucose deprivation-induced mitochondrial dysfunction and cell damage. Thus, the neuroprotective effects of magnolol could be related to its antioxidative activity and antagonism of excitotoxicity induced by excitatory amino acids. In conclusion, magnolol could be a potential therapeutic molecule against the neurodegenerative diseases.

There is a high interest for natural molecules against aging. Therefore, magnolol is one of the bioactive compounds from natural resources against skin aging [18]. Skin aging involves the degradation of extracellular matrix (epidermal and dermal layers) with visible consequences in the skin surface and modifications of the skin physical properties. The importance of these molecules has implications in the treatment of chronological and premature skin aging. In addition to the magnolol, there are other phytomolecules for this task: aloin, ginsenoside, curcumin, epicatechin, asiaticoside, ziyuglycoside I, gallic acid, hydroxychavicol, hydroxycinnamic acids, hydroxybenzoic acids, etc. [18]. These molecules are scavenges of free radicals or they are preventing the trans-epidermal water loss, protecting the skin from wrinkles and keeping a younger skin.

It is noteworthy that the relevant magnolol studies have mainly focused on the evaluation of its beneficial effects against oxidative radicals in vitro. However, few studies have been conducted to comprehensively investigate the effects of magnolol on the antioxidant enzyme system in relation to the oxidative stress in vivo. Consequently, the objectives of this study were to determine the anti-oxidative effects of magnolol on oxidative status, antioxidant enzyme activities, and antioxidant enzyme mRNA and protein expression in the livers of Enterotoxigenic Escherichia coli (ETEC)-induced and non-ETEC-induced mice. 


\section{MATERIALS AND METHODS}

\subsection{Experimental Methods}

\subsubsection{Ethics Statement}

The administration of mice and all experimental procedures were carried out in accordance with the Laboratory Animal Ethical Commission and the Ethics Committee of the Institute of Subtropical Agriculture, Chinese Academy of Sciences (No. 015063506). All mice were slaughtered after anesthetizing with sodium pentobarbital, and all procedures were done within the minimize suffering.

\subsubsection{Extracts and Reagents}

The commercial magnolol power (extracted from the cortex of Magnolia officinalis, purity > $98.0 \%$ in dry) was purchased from Jinnong Bio Co. Ltd. (Changsha, China) and kept at $-20^{\circ} \mathrm{C}$. The ETEC $\mathrm{O}_{78}: \mathrm{K}_{80}(44813)$ suspensions $(3.45 \times 109 \mathrm{CFU} / \mathrm{ml})$ was prepared in accordance with our previous report [44], and cultured overnight in lysogeny broth (LB) medium in a shaking incubator at $180 \mathrm{rpm}$ and $37^{\circ} \mathrm{C}$ for $12 \mathrm{~h}$. The cell density of ETEC suspension was measured by ND-1000 UV-vis spectrophotometer (Nano- Drop Ltd., TX).

\subsubsection{Experimental Design}

The experimental animals made use of eighty BALB/c female mice with the ages of $7-8$ weeks and the average body weight of $20-22 \mathrm{~g}$, purchased from Shanghai Laboratory Animal Center, the Chinese Academy of Science (SLACCAS, Shanghai, China). In the pre-experimental period, all mice were individually kept in a specific pathogen-free mouse colony in a standard environmental condition with the temperature of $20-30^{\circ} \mathrm{C}$, relative humidity of $40-60 \%$, and the light control cycle (illumination/dark) of $12 \mathrm{~h} / 12 \mathrm{~h}$ for 7 days. In addition, all mice had free access to autoclaved feed and sterile water.

All animals (80 mice) were randomly divided into eight groups $(\mathrm{n}=10 \mathrm{mice} / \mathrm{group})$, including the group was or was not orally administered with $0.02 \mathrm{ml} / \mathrm{g}$ ETEC strain suspension, after the oral administration of magnolol for 7 days for all groups, at the dosages of $0,100,300$, and $500 \mathrm{mg} / \mathrm{kg}$ mouse BW, respectively. The commercial magnolol power was equally dissolved in $0.4 \mathrm{ml} 2 \%$ Tween 80 solution before oral administration by gavage for each mouse. The ETEC suspension was prepared according to our previous report [44], and the density was measured with ND-1000 UV-vis spectrophotometer (NanoDrop Ltd., TX) at optical density 600 (OD 600=1 represents $1 \times$ $10^{10} \mathrm{CFU} / \mathrm{ml}$ cell density). In the present study, the determined cell density of ETEC suspension was $3.45 \times 10^{9} \mathrm{CFU} / \mathrm{ml}$. For the magnolol control groups, NC, M100, M300, M500 and ETEC, ME100, ME300, and ME500 represent whether the groups were or were not orally administered with ETEC suspension after orally administered with magnolol power at the dosages of 0, 100, 300 , and $500 \mathrm{mg} / \mathrm{kg} \mathrm{BW}$, respectively. Among, "M" represents the mice in a group were orally administered with magnolol solution, "E" represents the mice were intraperitoneally administered ETEC suspension $(0.02 \mathrm{ml} / \mathrm{g} \mathrm{BW})$. Formal test period, all mice were orally administered with magnolol solution for 7 days according to the magnolol dosage of each group. All mice were fasted for 6 hours. After this time, an intraperitoneally ETEC suspension was administered. The animals in all groups were sacrificed under sodium pentobarbital anesthesia for 3 hours after the administration of ETEC suspension. 


\subsubsection{Biochemical Analysis}

The liver tissue was sampled from each mouse and homogenized $(1 \mathrm{~g} / 10 \mathrm{ml}$ ice-cold $0.9 \%$ sterile saline) on ice. The homogenized solution was centrifuged at $3,500 \times \mathrm{g}$ for $15 \mathrm{~min}$ at $4^{\circ} \mathrm{C}$, and the supernatant was stored at $-20^{\circ} \mathrm{C}$ for further analysis. Nitric oxide (NO), malondialdehyde (MDA), myeloperoxidase (MPO), total antioxidant capacity (T-AOC), glutathione (GSH), catalase (CAT), superoxide dismutase (SOD), and glutathione peroxidase (GPx) in the supernatant of live samples were measured using spectrophotometric kits (A012, A003-1, A044, A015, A061, A0071, A001-1, and A005) in accordance with the manufacturer's instructions (Nanjing Jiancheng Bioengineering Institute, Nanjing, China) [14, 45].

\subsubsection{Real-time Quantitative RT-PCR}

Total RNA from the mouse livers was isolated using TRIzol reagent (Invitrogen, CA, USA) $\Delta$ according to the manufacturer's instructions within minor modification [44]. The concentration of isolated total RNA was quantified with an ND-1000 UV-vis spectrophotometer. Then, $1 \mu \mathrm{g}$ of RNA was treated with RNase-free DNase I to remove DNA and cDNA was synthesized using a PrimeScript ${ }^{\mathrm{TM}}$ RT reagent kit with gDNA Eraser (Takara Bio. Inc., Shiga, Japan). Primers (Table 1) were designed using Primer 5.0 software (Premier Co., Canada) according to the mouse gene sequences. The glyceraldehyde 3-phosphate dehydrogenase (GAPDH) was used as an internal control to normalize target gene transcript levels. Real-time PCR was performed with $\mathrm{SYBR}^{\mathrm{R}}$ Premix Ex Tap ${ }^{\mathrm{TM}}$ II (Tli RNaseH Plus) kits according to the manufacturer's instructions [44]. Briefly, $1 \mu \mathrm{l}$ cDNA template was added to a total volume of $10 \mu \mathrm{l}$ containing $5 \mu \mathrm{l} \mathrm{SYBR}$ Green mix, $0.2 \mu \mathrm{l}$ Rox, $3 \mu \mathrm{l}$ DEPC-treated distilled water, and $0.4 \mu \mathrm{l}$ each of the forward and reverse primers $(10 \mathrm{nM})$. Reactions were conducted in an ABI 7900HT fast real-time qRT-PCR system using the following protocol: $95^{\circ} \mathrm{C}$ for $30 \mathrm{~s}$ for pre-denaturation, followed by 40 cycles of $95^{\circ} \mathrm{C}$ for $5 \mathrm{~s}$ and $60^{\circ} \mathrm{C}$ for $30 \mathrm{~s}$ for amplification and quantification. To generate a melting curve, the temperature increased from $60^{\circ} \mathrm{C}$ to $95^{\circ} \mathrm{C}$ at a rate of $0.5^{\circ} \mathrm{C}$ over $10 \mathrm{~s}$. At the end of the real-time PCR, the threshold cycle value (CT) for each reaction was provided, and the change in the transcriptional level of the target gene normalized to GAPDH was calculated by the following formula: relative mRNA level of target gene (folds of control) $=2-(\Delta \Delta \mathrm{CT})$, where $\Delta \Delta \mathrm{CT}=(\mathrm{CT}$ Target - CT GAPDH) treatment - (CT Target - CT GAPDH) control.

Table 1. Sequences of primers (forward, for; reverse, rev), and size of real-time quantitative PCR products.

\begin{tabular}{lcccc}
\hline Gene $^{1}$ & $\begin{array}{c}\text { Accession } \\
\text { No. }\end{array}$ & For Primers (5'-3') & Rev Primers (5'- 3') & $\begin{array}{c}\text { Product Size } \\
(\mathrm{bp})\end{array}$ \\
\hline GAPDH & NM_008084 & GACTCCACTCACGGCAAATTCA & TCGCTCCTGGAAGATGGTGAT & 93 \\
CAT & NM_009804 & CGTTCGATTCTCCACAGTCA & CCCACAAGATCCCAGTTACC & 111 \\
CuZn- & NM_011434 & CGGATGAAGAGAGGCATGTT & GTACGGCCAATGATGGAATG & 124 \\
SOD & & & & \\
MnSOD & NM_013671 & CAAGCGTGACTTTGGGTCTT & GCGACCTTGCTCCTTATTGA & 112 \\
ECSOD & NM_011435 & TGTTCTACGGCTTGCTACTGG & ATGCGTGTCGCCTATCTTCT & 143 \\
GPx-1 & NM_008160 & AAGGCTCACCCGCTCTTTAC & ACACCGGAGACCAAATGATG & 106 \\
& & & & \\
\hline
\end{tabular}

${ }^{1} \mathrm{GAPDH}=$ glyceraldehyde-3-phosphate dehydrogenase CAT = catalase CuZn-SOD = superoxide dismutase $1 ; \mathrm{MnSOD}=$ superoxide dismutase $2 ; \mathrm{ECSOD}=$ superoxide dismutase $3 ; \mathrm{GPx}-1=$ glutathione peroxidase 1 . 


\subsubsection{Western Blot Analysis}

The total protein was extracted from the liver tissue using a total protein extraction kit, and the protein concentration was estimated using an enhanced Bicinchoninic Acid (BCA) protein assay kit (Beyotime Institute of Biotechnology Inc., Jiangsu, China). Equivalent quantities of protein were denatured at $100^{\circ} \mathrm{C}$ for $7 \mathrm{~min}$ and were separated by reducing $10 \%$ SDS-PAGE electrophoresis purchased from Beyotime Institute of Biotechnology Inc. The isolated proteins were transferred onto PVDF membranes (Millipore, MA, USA) with $0.45 \mu \mathrm{m}$ apertures at a constant $200 \mathrm{~mA}$ for $70 \mathrm{~min}$. The nonspecific binding sites of the membranes were blocked with $5 \%$ non-fat milk in Tris-Tween buffered saline for 2 hours. The primary antibodies for SOD, CAT, GPx-1 and $\beta$-actin (Abcam Com., UK) were incubated with the membranes overnight at $4^{\circ} \mathrm{C}$. Thereafter, they were subsequently incubated with horseradish peroxidase-conjugated secondary antibodies for 1 hour at room temperature before detection was performed by chemiluminescent reaction with Luminata TM Crescendo Western HRP Substrate (Merck Millipore, USA) Densitometric signals were obtained after the membranes were exposed to an X-ray film. The band intensities were quantified by Quantity One software (Bio-Rad) and normalized versus $\beta$ actin as an internal control for total protein loading.

\subsection{Computational Methods}

In the theoretical study, a unique QSAR classification model has been developed using different types of Machine Learning techniques from Weka software [46]. The best model is able to predict magnolol ChEMBL activities for specific protein targets and organisms. The final model features are encoding the chemical information using molecular descriptors.

The steps to find the best classification models for magnolol interactions prediction are the followings:

1. Download of the initial dataset - from ChEMBL website, complete data was downloaded for all the interactions between the compounds and magnolol experimental targets (biological activity type, units of activity, SMILES formula for the compounds, protein target, organism);

2. Create the QSAR model dataset:

a. Calculation of 62 descriptors of the ChEMBL compounds using Rcpi package [47] from $\mathrm{R}$ programming language [48].

b. Calculation of moving averages (MAs) for all descriptors using 3 experimental conditions: the type \& units of the ChEMBL activity, protein target and organism. MAs are differences between the initial descriptors and the average values of the descriptors for a specific experimental condition. Thus, the final dataset has 186 features as MAs of the compound's descriptors (62 descriptors $* 3$ experimental conditions).

c. Search for the best QSAR model classification model based on compounds interactions with protein targets of magnolol using six Machine Learning techniques from Weka software: LibLINEAR [49], BayesNet [50], DecisionTable [51], RandomTree [52], J48 [53], and RandomForest [54]. The criteria for the best model is the maximum true positive rate (TPR) and Area Under Receiver Operating Characteristic (AUROC) [55].

d. Prediction of 79 ChEMBL activities of magnolol with 47 types of proteins in 18 organisms (Weka software). 
Let's consider for each $i$ compound that the output activity with specific units is ${ }^{\text {unit }} \mathrm{A}_{\mathrm{i}}$ and $j$ molecular descriptors $(j=1-62)$ in specific experimental conditions (activity type, protein target and organism) are exper $\mathrm{D}_{\mathrm{ij}}$. The corresponding moving averages of the descriptors in three experimental conditions are ${ }^{\text {activity }} \mathrm{MAD}_{\mathrm{ij}}$, ${ }^{\text {target }} \mathrm{MAD}_{\mathrm{ij}}$ and ${ }^{\text {organism }} \mathrm{MAD}_{\mathrm{ij}}\left[{ }^{\text {exper }} \mathrm{MAD}={ }^{\text {exper }} \mathrm{D}-\right.$ average(D)exper]. Thus, the general linear classifier Class $\mathrm{i}_{\mathrm{i}}$ could be described by Eq. $1\left(\mathrm{a}_{\mathrm{ij}}, \mathrm{b}_{\mathrm{ij}}, \mathrm{c}_{\mathrm{ij}}\right.$ and $\mathrm{a}_{0}=$ linear coefficients).

$\operatorname{Class}_{i}^{+/-}\left({ }^{\text {exper }} A_{i}\right)=\sum_{j=1}^{62} a_{i j}$.activity $\mathrm{MAD}_{i j}+\sum_{j=1}^{62} b_{i j} \cdot{ }^{\text {target }} \mathrm{MAD}_{i j}+\sum_{j=1}^{62} c_{i j}$. organism $\mathrm{MAD}_{i j}+\alpha_{0}$

The initial activity values has been transformed into positive and negative classes as following: if the difference between the activity value of one case and the average values of the activity in the dataset is greater than zero, the class is positive (otherwise the class is negative). Thus, the QSAR classification model is able to predict of the values of the new compound activities are greater and lower than the average value of the activity in the dataset.

The final dataset contains 38,468 cases with 186 features as ${ }^{\text {exper }}$ MADij (moving averages for each molecular descriptor in specific experimental condition). The default crossvalidation type in Weka was used (10-fold cross-validation). The best model was the classification model with maximum TPR and AUROC.

The model could be used to predict for any compound with a SMILES formula the ChEMBL activity level for specific protein target and organism. Because the current experimental study was focused on magnolol, the QSAR model was used to complete these experiments by prediction of all activity types with all possible targets and organisms for magnolol.

\section{RESULTS AND DISCUSSION}

\subsection{Experimental Study of ROS Activity of Magnolol}

\subsubsection{Experimental Results}

The oxidative status of the livers of the mice was assessed by biochemical parameters, including MDA, MPO, NO, T-AOC and GSH (Fig. 1). Compared with the NC treatment, intraperitoneal administration of the ETEC suspension increased $(P<0.05)$ the production of MDA (Fig. 1A), MPO (Fig. 1B), NO (Fig. 1C) and T-AOC (Fig. 1D), but reduced $(P<0.05)$ the GSH level (Fig. 1E) in the livers of mice. Oral administration of magnolo at $100 \mathrm{mg} / \mathrm{kg} \mathrm{BW}$ for 7 days decreased $(P<0.05)$ the MPO level, and at 300 and $500 \mathrm{mg} / \mathrm{kg} \mathrm{BW}$, it increased $(P<0.05)$ the NO and GSH levels in the livers of the non-ETEC-induced mice. Compared with ETEC induction alone, magnolol pretreatment at a dose of $500 \mathrm{mg} / \mathrm{kg} \mathrm{BW}$ significantly reduced $(P<$ $0.05)$ the MPO level, and magnolol pretreatment at all doses decreased $(P<0.05)$ the NO and TAOC levels in the livers of the ETEC-induced mice. 

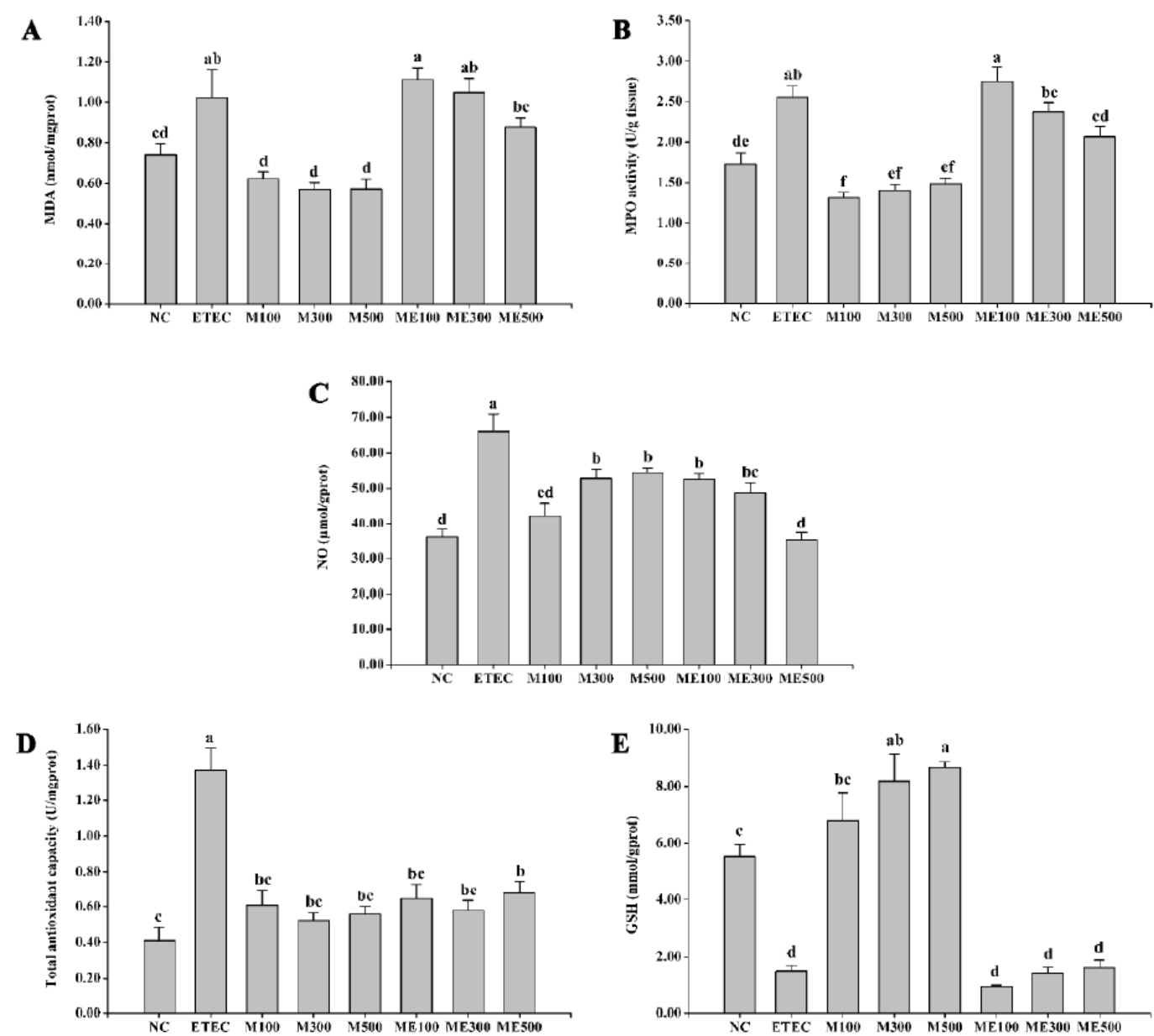

Fig. (1). Concentrations of MDA (A), MPO (B), NO (C), T-AOC (D) and GSH (E) in non-ETEC-induced and ETECinduced mice. Values are the mean \pm SEM. The values with different superscript letters significantly differ $(P<0.05)$. NC = normal control; ETEC $=$ Enterotoxigenic Escherichia coli suspension-induced control; M100, M300, M500 = oral administration of magnolol at doses of 100, 300 and $500 \mathrm{mg} / \mathrm{kg} \mathrm{BW}$; ME100, ME300, ME500 = oral administration of magnolol at doses of 100, 300 and $500 \mathrm{mg} / \mathrm{kg} \mathrm{BW}$ and then induction with the Enterotoxigenic Escherichia coli suspension.

Compared with the NC group, SOD activity (Fig. 2A) and the mRNA expression of ECSOD (Fig. 2D) was significantly reduced $(P<0.05)$ in the mice that received intraperitoneal administration of the ETEC suspension, although the MnSOD (Fig. 2C) mRNA expression was dramatically increased $(P<0.05)$. Interestingly, the higher doses of magnolol treatment did not promote and instead inhibited $(P<0.05)$ SOD activity $(500 \mathrm{mg} / \mathrm{kg}$ BW, Fig. 2A) and ECSOD mRNA expression (300 and $500 \mathrm{mg} / \mathrm{kg} \mathrm{BW}$, Fig. 2D), but the lower doses (100 and $300 \mathrm{mg} / \mathrm{kg}$ ) of magnolol treatment markedly promoted $(P<0.05)$ CuZn-SOD mRNA expression (Fig. 2B). Compared with that in the ETEC group, CuZn-SOD mRNA expression was increased $(P<0.05)$ in the livers of the ETEC-induced mice that received magnolol pretreatment at doses of 100 and $500 \mathrm{mg} / \mathrm{kg} \mathrm{BW}$ (Fig. 2B), while ECSOD mRNA expression was increased in those that received doses of 300 and $500 \mathrm{mg} / \mathrm{kg} \mathrm{BW}$ (Fig. 2D). 

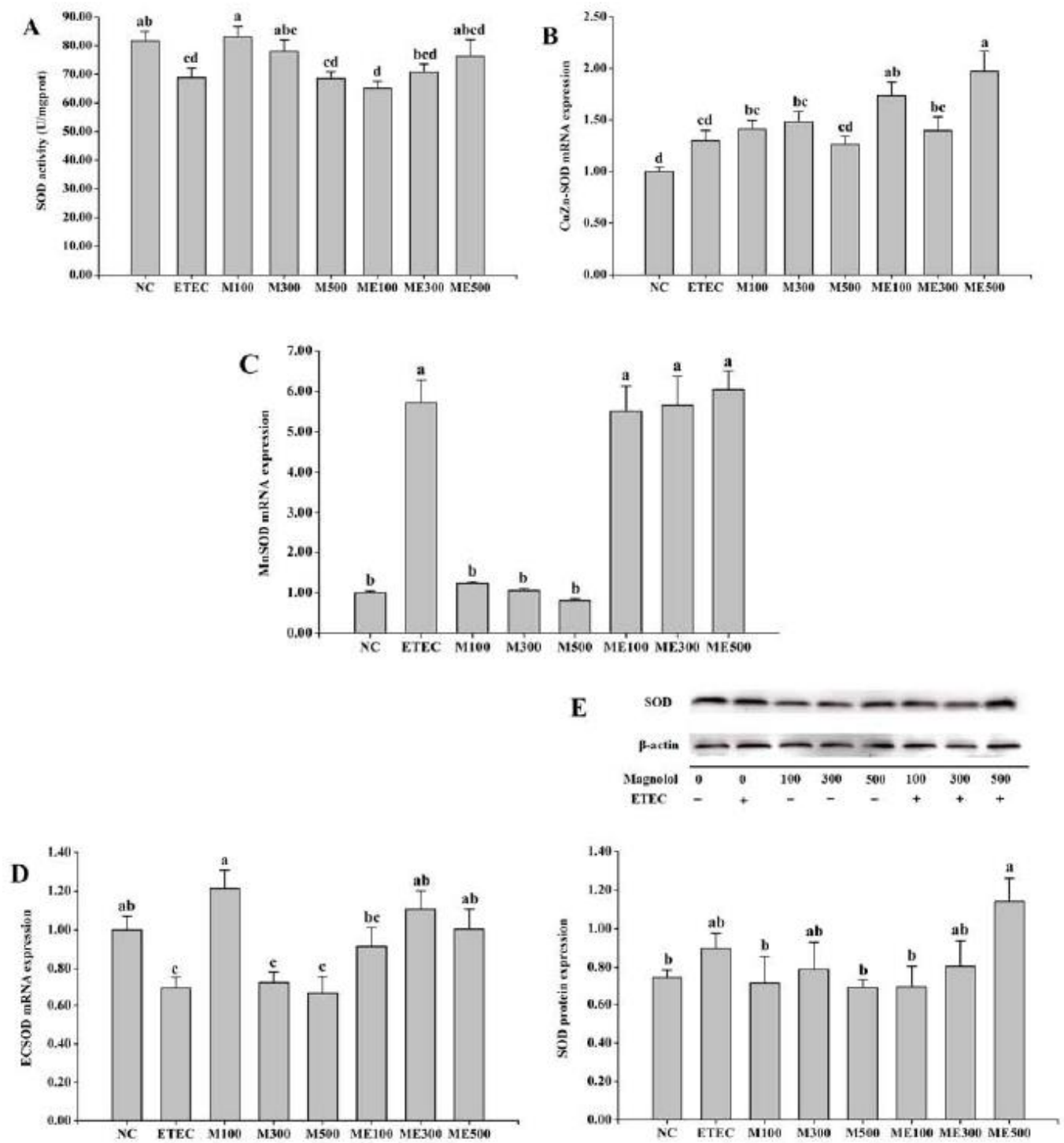

Fig. (2). Effects of orally administered magnolol on SOD activity and relative SOD mRNA and protein expression in non-ETECinduced and ETEC-induced mice. Values are the mean \pm SEM $(\mathrm{n}=10)$. The values with different superscript letters significantly differ $(P<0.05)$. NC $=$ normal control; ETEC $=$ Enterotoxigenic Escherichia coli suspension-induced control; M100, M300, M500 = oral administration of magnolol at doses of 100, 300 and $500 \mathrm{mg} / \mathrm{kg} \mathrm{BW;} \mathrm{ME100,} \mathrm{ME300,}$ ME500 = oral administration of magnolol at doses of 100,300 and $500 \mathrm{mg} / \mathrm{kg} \mathrm{BW}$ and then induction with the Enterotoxigenic Escherichia coli suspension.

Compared with the NC treatment, the intraperitoneal administration of ETEC suspension or oral administration of magnolol did not affect $(P>0.05)$ the GPx activity, mRNA and protein expression in the livers of the ETEC-induced or non-ETEC-treated mice (Fig.3A, B and $\mathbf{C}$ ), respectively. Compared to the corresponding levels in the ETEC group, GPx activity and mRNA expression were increased $(P<0.05)$ in the livers of the ETEC-induced mice that received magnolol pretreatment at $500 \mathrm{mg} / \mathrm{kg} \mathrm{BW}$ (Fig. 3A and B). Additionally, there was a slight increase in GPx protein expression only in the ETEC-induced mice that received the $500 \mathrm{mg} / \mathrm{kg} \mathrm{BW}$ magnolol treatment (Fig. 3C). 

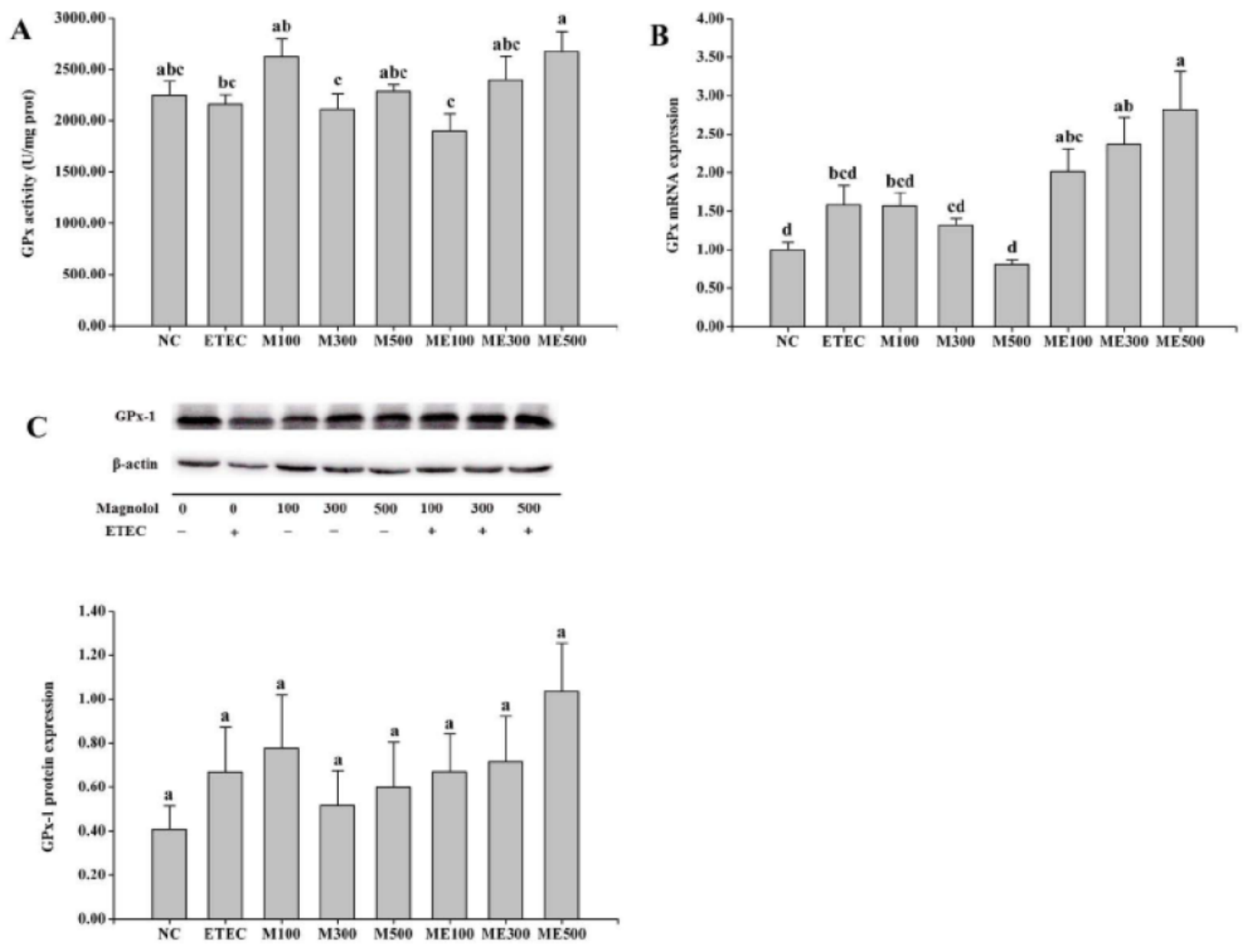

Fig. (3). Effects of orally administered magnolol on GPx activity and relative GPx-1 mRNA and protein expression in non-ETECinduced and ETEC-induced mice. Values are the mean - \pm SEM $(\mathrm{n}=10)$. The values with different superscript letters significantly differ $(P<0.05) . \mathrm{NC}=$ normal control; ETEC $=$ Enterotoxigenic Escherichia coli suspension-induced control; M100, M300, M500 = oral administration of magnolol at doses of 100, 300 and $500 \mathrm{mg} / \mathrm{kg}$ BW; ME100, ME300, ME500 = oral administration of magnolol at doses of 100,300 and $500 \mathrm{mg} / \mathrm{kg}$ BW and then induction with the Enterotoxigenic Escherichia coli suspension.

Compared with the NC treatment, the intraperitoneal administration of the ETEC suspension reduced $(P<0.05)$ the activity and mRNA expression of CAT (Fig. $\mathbf{4 A}$ and $\mathbf{B}$ ); however, the ETEC suspension infection did not affect CAT protein expression (Fig. 4C). Magnolol treatment at 300 and $500 \mathrm{mg} / \mathrm{kg} \mathrm{BW}$ suppressed $(P<0.05)$ CAT activity but at $100 \mathrm{mg} / \mathrm{kg} \mathrm{BW}$, it promoted $(P<0.05)$ CAT mRNA expression in the livers of the non-ETEC-induced mice (Fig. 4A and $\mathbf{B})$. Moreover, compared with ETEC induction alone, magnolol pretreatment at most levels (100, 300 and $500 \mathrm{mg} / \mathrm{kg} \mathrm{BW}$ ) decreased $(P<0.05)$ the activity and mRNA expression of CAT (Fig. 4A and B). Subsequently, CAT protein expression (Fig. 4C) was not affected by magnolol treatment, regardless of whether the mice were treated with ETEC. 
A

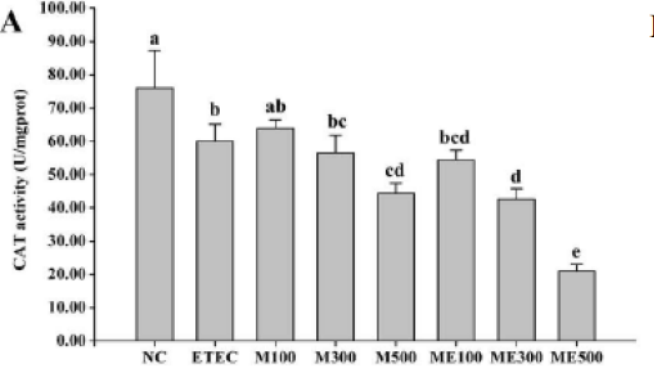

C
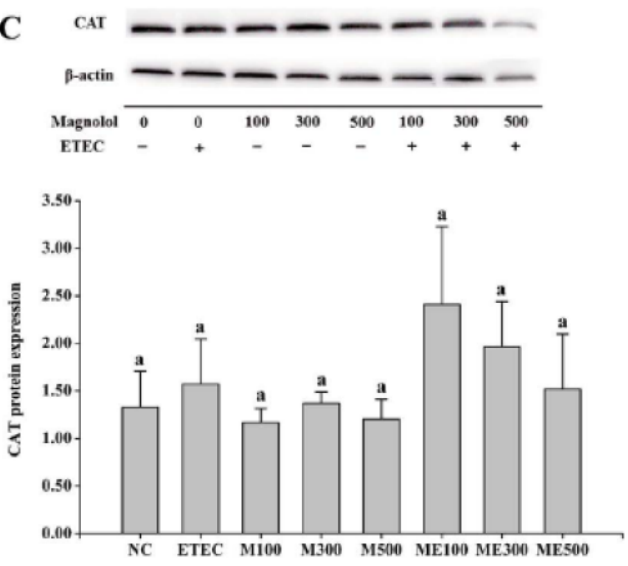

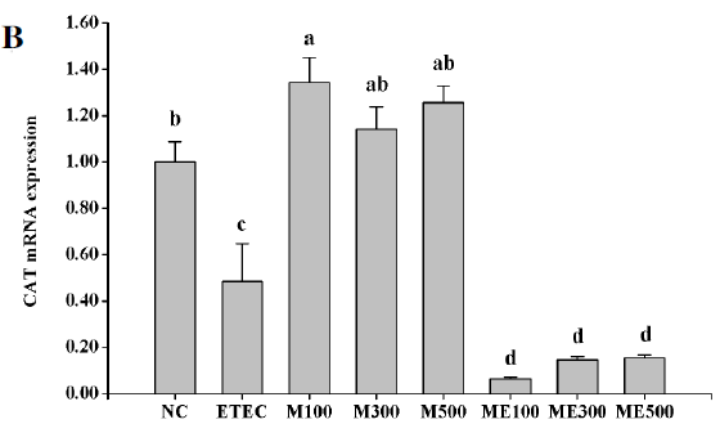

Fig. (4). Effects of orally administered magnolol on CAT activity and relative CAT mRNA and protein expression in non-ETECinduced and ETEC-induced mice. Values are the mean $\bullet \pm$ SEM $(\mathrm{n}=10)$. The values with different superscript letters significantly differ $(P<0.05) . \mathrm{NC}=$ normal control; ETEC = Enterotoxigenic Escherichia coli suspension-induced control; M100, M300, M500 = oral administration of magnolol at doses of $100,300 \mathrm{and} 500 \mathrm{mg} / \mathrm{kg}$ BW; ME100, ME300, ME500 = oral administration of magnolol at doses of 100, 300 and $500 \mathrm{mg} / \mathrm{kg} \mathrm{BW}$ and then induction with the Enterotoxigenic Escherichia coli suspension.

\subsubsection{Discussion of Magnolol Activity}

In bio-systems, oxidative stress generally results from an imbalance between the pro-oxidative and anti-oxidative systems [1]. Previous studies have demonstrated that infection with enteric bacterial pathogens or the lipopolysaccharide induces inflammation [56], tissues damage and apoptosis and causes oxidative stress [57]. Several end products of lipid peroxidation have been used as biomarkers of oxidative stress, including MDA, NO and MPO [58, 59]. Total antioxidant capacity and GSH reflect the capacity of the nonenzymatic antioxidant defense system [59]. In this study, administration of the ETEC suspension increased the concentration of MDA, MPO, NO, and T-AOC, but reduced liver GSH levels in the liver tissue, further indicating that ETEC infection partly caused liver damage and induced lipid peroxidation, indicating that the livers of the experimental mice may be experiencing oxidative stress.

It has been reported that magnolol can decrease MDA concentrations in rat ileum after lipopolysaccharide injection [57] or in diabetic rats liver [11]. This supported the current results where magnolol reduced the MDA concentrations in the livers of mice with ETEC induction. The current results showed that magnolol treatment reduced MPO levels in mice with or without ETEC induction, in agreement with the previous report [12]. Interestingly, the increase in NO caused by higher doses of oral magnolol may be related to the prooxidative capacity of magnolol in nonETEC-induced mice; these results are partly consistent with the previous findings, in which a significant pro-oxidative effect could be easily triggered by treatment with higher doses of flavonoid or vitamin E $[60,61]$. In oxidative stress, magnolol can effectively reduce NO levels in the rat hind limb muscle model [12] which supported the results of the magnolol pretreatment at 
all of the administered doses reducing NO levels in ETEC-induced mice. Of note, the T-AOC levels were reduced by oral magnolol in the ETEC-induced mice, while our previous study reported that magnolol treatment increased liver T-AOC levels in physiologically normal mice [14]. The possible explanations were that magnolol may restore the T-AOC levels in the bacteria induced oxidative stress. GSH levels were continuously increased by oral magnolol at different doses in the non-ETEC-induced mice supporting our previous report [14]. The previous study has demonstrated that magnolol treatment alleviated the GSH depletion in rat liver tissue induced by acetaminophen [9]. Pretreatment with quercetin for 15 days resulted in a significant increase in the hepatic GSH concentration, but the protective effects were not observed when the mice were treated with quercetin after chronic exposure to ethanol [62]. Some studies have indicated that GSH has the capacity to directly scavenge hydroxyl radical and singlet oxygen; acting as a cofactor of several detoxifying enzymes, glutathione peroxidase, and others; participating in amino acid transport; and regenerating the most important antioxidants (e.g., vitamins $\mathrm{C}$ and $\mathrm{E})$ [1, 63]. It was observed that magnolol did not restore the GSH depletion in ETEC-induced mice. To some extent, we propose that magnolol has unique effects on NO, T-AOC and GSH activity under normal physiological or pathological conditions due to their multiple effects in all of the lines of protection against oxidative stress [3].

Obviously, the present study revealed some novel evidences that magnolol played a different role in modulating the relevant antioxidant enzymes. For instance, magnolol raised the mRNA expression of soluble superoxide dismutase 1 (CuZn-SOD), but reduced SOD activity and the mRNA expression of extracellular superoxide dismutase 3 (ECSOD) in non-ETEC-induced mice. Interestingly, these results were partly different with our previous results that the mRNA expression of CuZn-SOD and superoxide dismutase 2 or mitochondrial (MnSOD) were not altered after 14 days of magnolol administration in Kunming mice, although total SOD activity and ECSOD mRNA expression were increased [14]. Simultaneously, administration of the ETEC suspension reduced total SOD activity and ECSOD mRNA expression but increased MnSOD mRNA expression. However, magnolol had significant antioxidant effects on total SOD activity or mRNA (e.g., CuZn-SOD and ECSOD) and protein expression in ETEC-induced mice which was in agreement with the previous reports [7, 11, 57]. Based on the previous studies of flavonoids and polyphenols, it might be surmised that the effects of antioxidants depend on different factors, such as the specific compound used, the cell type, the dose, and the experimental design [3, 4, 60, 61]. Therefore, based on this fact together with the data of SOD activity and mRNA and protein expression, we suggest that magnolol played a different role in SOD-mediated enzymatic antioxidant defense against superoxide radicals.

The low oral dose of magnolol caused a numerical increment in hepatic antioxidant enzymatic activity and the mRNA and protein expression of GPx; these effects were partially validated by our previous report in the physiological mouse liver [14]. In contrast, oral magnolol $500 \mathrm{mg} / \mathrm{kg}$ BW predominantly increased the antioxidant activity and mRNA expression of GPx in ETECinduced mice. To some extent, these results do not conflict with the former report, in which the oral magnolol significantly increased the hepatic antioxidant activity of GPx in diabetic rats [11]. A reasonable explanation is that the antioxidant capacity of magnolol in different pathophysiological conditions depends on the dose.

CAT has been shown to be regulated at multiple levels by diverse polyphenols. For instance, feeding rats a $2 \%$ garlic diet for 2 weeks reduced CAT activity and its protein expression level, whereas the CAT mRNA level remained unchanged in the kidney and liver [64]. Moreover, resveratrol was shown to inhibit the mRNA expression of CAT in rotifers [65]. In addition, oral administration of magnolol significantly increased the hepatic activity of CAT in the livers of diabetic rats [11] and increased the activity and mRNA level of CAT in normal mice [14]. Oral magnolol decreased the activity of CAT in ETEC-induced or non- ETEC-induced mice, while it increased the CAT mRNA level in non-ETEC-induced mice, but decreased CAT mRNA and protein expression in a dose-dependent manner in ETEC-induced mice. This evidence suggests 
that CAT could be modulated and controlled by magnolol, an effect that distinguishes it from other polyphenols, in a manner strongly dependent on the dose and the pathophysiological condition.

\subsection{QSAR Model for Magnolol ChEMBL Protein Targets}

The experimental results have been completed with computational study for the evaluation of the interactions of magnolol by different ChEMBL activities with different protein targets in organisms. The main dataset was obtained for the molecules with the activities against magnolol protein targets, in specific organisms. This data was completed with the molecular Rcpi descriptors.

Moving averages of these descriptors for types of activities, protein targets and organism were the final features of the models. The output values of activities were transformed in two classes using the averages values of each activity type: positive class for the cases where the values are greater than the averaged one and negative class for opposite cases. The prediction is using Machine Learning classifiers that are able to predict if a specific ChEMBL activity of magnolol is greater or less than the average value of the dataset activities.

Six Machine Learning classification methods were tested by Weka software: LibLINEAR, BayesNet, DecisionTable, RandomTree, J48, and RandomForest. The values of TPR and AUROC for each classifier are presented in Table 2. The results show that linear models are not enough to describe the relation between the output and the features. Bayes Nets gives better results but it has a very low performance. The rules method such as Decision Table is slowly improving the classification performance compared with Bayes Net but with TPR $<0.64$ and AUROC $<0.71$. The next three methods are based on trees: Random Tree, J48 and Random Forest. The results demonstrate that the methods based on one single tree (Random Tree and J48) can improve TPR and AUROC but only multiple trees (Random Forest) are able to provide weighted TPR $>0.70$ and AUROC $>0.79$. Additional test have been done for different numbers of trees for RF method. Improvements of TPR \& AUROC can be observed from 100 trees to 500 trees but almost no improvement was obtained with 1,000 trees.

Table 2. Machine Learning classification models for interaction of magnolol with protein targets

\begin{tabular}{lcc}
\hline ML Method & TPR & AUROC \\
\hline LibLINEAR & 0.528 & 0.524 \\
BayesNet & 0.609 & 0.686 \\
DecisionTable & 0.632 & 0.707 \\
RandomTree & 0.649 & 0.637 \\
J48 & 0.661 & 0.721 \\
RandomForest & 0.701 & 0.791 \\
\hline
\end{tabular}

Therefore, the best model that can predict the magnolol interactions characterized by a specific biological activity with protein targets in specific organisms was obtained by Random Forest method using 500 trees. The model has the weighted TPR of 0.701 and AUROC of 0.790 . 
The best model was used to predict the interactions using 79 types of ChEMBL biological activities for 47 protein targets and 18 organisms. Table 3 presents the predicted interactions greater than the averaged ones in the dataset with protein targets in Bos taurus, Homo sapiens, Mus musculus, Rattus norvegicus and Schistosoma mansoni (probability > 0.60). The entire list of magnolol predictions for all possible experimental combinations is presented as Supplementary Material.

Table 3. Prediction of magnolol interactions with protein targets in Homo sapiens is greater than the average ones in the ChEMBL dataset.

\begin{tabular}{lccc}
\hline Organism & Standard Type & Units & Target Name \\
\hline & & & \\
Bos taurus & Inhibition & $\%$ & Glutathione S-transferase A1 \\
Homo sapiens & Activity & $\%$ & Prostaglandin E synthase \\
Homo sapiens & Inhibition & $\%$ & Glutathione reductase homolog \\
Homo sapiens & Inhibition & $\%$ & Glutathione reductase, putative \\
Homo sapiens & Inhibition & $\%$ & Glutathione S-transferase \\
Homo sapiens & Inhibition & $\%$ & Glutathione S-transferase A1 \\
Homo sapiens & Inhibition & $\%$ & Probable glutathione reductase 2 \\
Homo sapiens & Inhibition & $\%$ & Prostaglandin E synthase \\
Homo sapiens & Inhibition & $\%$ & S-formylglutathione hydrolase \\
Homo sapiens & Inhibition & $\%$ & Superoxide dismutase [Cu-Zn] \\
Mus musculus & Inhibition & $\%$ & Glutathione reductase, putative \\
Rattus norvegicus & Activity & $\%$ & Prostaglandin E synthase \\
Rattus norvegicus & Inhibition & $\%$ & Esterase D \\
Rattus norvegicus & Inhibition & $\%$ & Glutathione reductase, putative \\
Rattus norvegicus & Inhibition & $\%$ & Glutathione S-transferase \\
Rattus norvegicus & Inhibition & $\%$ & Glutathione S-transferase A1 \\
Rattus norvegicus & Inhibition & $\%$ & Glutathione synthetase \\
Rattus norvegicus & Inhibition & $\%$ & Probable glutathione reductase 2 \\
Rattus norvegicus & Inhibition & Prostaglandin E synthase \\
Rattus norvegicus & Inhibition & $\%$ & Superoxide dismutase [Cu-Zn] \\
Schistosoma mansoni & Potency & $\mathrm{nM}$ & Thioredoxin glutathione reductase \\
\hline
\end{tabular}

The magnolol experimental results in mouse were confirmed with the current theoretical model such as interactions with glutathione and superoxide dismutase. In addition, esterase D and prostaglandin E synthase are predicted as new targets. Similar with the experimental results in mouse, magnolol was predicted to have interactions in humans with glutathione and superoxide dismutase greater that the average interactions in the current dataset. In addition, new targets are predicted such as prostaglandin E synthase and Sformylglutathione hydrolase. The greater probability was obtained for the thioredoxin glutathione reductase in Schistosoma mansoni, a human parasite. Thus, the Random Forest model is confirming the most common protein target of magnolol among organisms as the glutathione reductase. The prediction model should be improved in future studies by using different types of information encoded in molecular descriptors. 


\section{CONCLUSION}

In the present study, the experiment strongly evidenced that the various levels of oral magnolol administration played a vital role against the expression and existence of superoxide radicals (e.g., the expressions of SOD, GPX-1, and CAT at the transcriptional and post-transcriptional levels) in the mouse enzymatic antioxidant defense system. On the other hand, the oral magnolol administration also influenced the enzyme activities (e.g., MDA, MPO, NO, T-AOC and GSH) in the mouse non-enzymatic antioxidant defense system. In addition, the maintenance of magnolol antioxidant capacity to protect organisms varied with its doses and the physiological status of the corresponding cells in the Enterotoxigenic E. coli (ETEC)-induced and non-ETEC-induced mice.

In order to complete the experimental results, a theoretical study predicted 79 types of biological activities of magnolol with 47 different protein targets in 18 organisms. The model dataset was based on the moving averages of the Rcpi molecular descriptors in specific experimental conditions such as activity type (with units), protein target and organism. Thus, six Machine Learning methods from Weka were tested to build the best classification model that can predict if the interactions of magnolol with protein targets is greater or less the average interactions of ChEMBL database. The best QSAR model was obtained with Random Forest method (10- fold cross-validation) and it has TPR of 0.701 and AUROC of 0.790 (test subset).

\section{CONSENT FOR PUBLICATION}

Not applicable.

\section{CONFLICT OF INTEREST}

The authors declare no conflict of interest, financial or otherwise.

\section{ACKNOWLEDGEMENTS}

This study was financially supported by the National Natural Science Foundation of China (Grant No. 30972166), Hunan Provincial Education Department (Grant No. 08A031), Hunan Provincial Innovation Foundation for Postgraduate (CX2011B304, CX2014B300). This work was supported by the "Galician Network for Colorectal Cancer Research (REGICC)" (ref R2014/039), funded by Xunta de Galicia. In addition, the current work was supported by the General Directorate of Culture, Education and University Management of Xunta de Galicia (Ref. GRC2014/049), Spain and finally by the Spanish Ministry of Economy and Competitiveness for its support with the funding of the unique installation BIOCAI (UNLC08-1E-002, UNLC13- 133503). L. A. Pastur-Romay thank the Principia program of Xunta de Galicia (Ref. IN848C_2016_1895718,09. A3.561A.480.0).

\section{SUPPLEMENTARY MATERIAL}

Supplementary material is available on the publishers web site along with the published article. 


\section{REFERENCES}

[1] Valko, M.; Leibfritz, D.; Moncol, J.; Cronin, M.T.D.; Mazur, M.; Telser, J. Free radicals and antioxidants in normal physiological functions and human disease. Int. J. Biochem. Cell Biol., 2007, 39(1), 44-84

[2] Galanos, C.; Freudenberg, M.A. Bacterial-endotoxins - Biological properties and mechanisms of action. Mediators Inflamm., 1993, 2, S11-S16.

[3] Masella, R.; Di Benedetto, R.; Vari, R.; Filesi, C.; Giovannini, C. Novel mechanisms of natural antioxidant compounds in biological systems: Involvement of glutathione and glutathionerelated enzymes. J. Nutr. Biochem., 2005, 16(10), 577-586.

[4] Rahman, I.; Biswas, S. K.; Kirkham, P.A. Regulation of inflammation and redox signaling by dietary polyphenols. Biochem. Pharmacol., 2006, 72(11), 1439-1452.

[5] Chen, Y.H.; Huang, P.H.; Lin, F.Y.; Chen, W.C.; Chen, Y.L.; Yin, W.H.; Man, K.M.; Liu, P.L. Magnolol: A multifunctional compound isolated from the Chinese medicinal plant Magnolia officinalis. Euro. J. Integrat. Med., 2011, 3(4), E311-E318.

[6] Yang, S.E.; Hsieh, M.T.; Tsai, T.H.; Hsu, S.L. Down-modulation of Bcl-X-L, release of cytochrome $\mathrm{c}$ and sequential activation of caspases during honokiol-induced apoptosis in human squamous lung cancer CH27 cells. Biochem. Pharmacol., 2002, 63(9), 1641-1651.

[7] Salto, J.; Shibuya, K.; Nagase, H. Anti-clastogenic effect of magnolol on benzo(a)pyreneinduced clastogenicity in mice. Food Chem. Toxicol., 2008, 46(2), 694-700.

[8] Fu, Y.H.; Liu, B.; Zhang, N.S.; Liu, Z. C.; Liang, D.J.; Li, F.Y.; Cao, Y.G.; Feng, X.S.; Zhang, X.C.; Yang, Z.T. Magnolol inhibits lipopolysaccharide-induced inflammatory response by interfering with TLR4 mediated NF-kappa B and MAPKs signaling pathways. $J$. Ethnopharmacol., 2013, 145(1), 193-199.

[9] Chen, Y.H.; Lin, F.Y.; Liu, P.L.; Huang, Y.T.; Chiu, J.H.; Chang, Y.C.; Man, K.M.; Hong, C.Y.; Ho, Y.Y.; Lai, M.T. Antioxidative and hepatoprotective effects of magnolol on acetaminopheninduced liver damage in rats. Arch. Pharm. Res., 2009, 32(2), 221-228.

[10] Saito, J.; Shibuya, K.; Nagase, H. Anti-clastogenic effect of magnolol on benzo(a)pyreneinduced clastogenicity in mice. Food Chem. Toxicol., 2008, 46(2), 694-700.

[11] Wang, J.J.; Zhao, R.; Liang, J.C.; Chen, Y. The antidiabetic and hepatoprotective effects of magnolol on diabetic rats induced by high-fat diet and streptozotocin. Yао Хие Хие Вао, 2014, 49(4), 476-481.

[12] Chen, H.Y.; Hung, Y.C.; Lee, E.J.; Chen, T.Y.; Chuang, I.C.; Wu, T.S. The protective efficacy of magnolol in hind limb ischemiareperfusion injury. Phytomedicine, 2009, 16(10), 976-981.

[13] Li, X.C.; Fang, Q.; Lin, J.; Yuan, Z.P.; Han, L.; Gao, Y.X. Chemistry study on protective effect against center dot $\mathrm{OH}$-induced DNA damage and antioxidant mechanism of cortex magnoliae officinalis. Bull. Korean Chem. Soc., 2014, 35(1), 117-122.

[14] Pang, Y.L.; Han, X.F.; Bamikole, M.A.; Gong, Z.H.; Tang, S.X.; Tan, Z.L.; Xiao, W.J.; Zhou, C.S.; Wang, M.; Deng, Y.L. Antidiarrhea and anti-oxidant properties of Magnolol. Trop. J. Pharmaceut. Res., 2013, 12(1), 85-91.

[15] Lo, Y.C.; Teng, C.M.; Chen, C.F.; Chen, C.C.; Hong, C.Y. Magnolol and honokiol isolated from Magnolia officinalis protect rat heart mitochondria against lipid peroxidation. Biochem. Pharmacol., 1994, 47(3), 549-553.

[16] Yao, K.; Zhang, L.; Ye, P.P.; Tang, X.J.; Zhang, Y.D. Protective effect of magnolol against hydrogen peroxide-induced oxidative stress in human lens epithelial cells. Am. J. Chin. Med., 2009, 37(4), 785-796.

[17] Lin, YR.; Chen, H.H.; Ko, C.H.; Chan, M.H. Neuroprotective activity of honokiol and magnolol in cerebellar granule cell damage. Eur. J. Pharmacol., 2006, 537(1-3), 64-69.

[18] Mukherjee, P.K.; Maity, N.; Nema, N.K.; Sarkar, B.K. Bioactive compounds from natural resources against skin aging. Phytomedicine, 2011, 19(1), 64-73.

[19] Zhang, S.; Golbraikh, A.; Tropsha, A. Development of quantitative structure-binding affinity relationship models based on novel geometrical chemical descriptors of the protein-ligand interfaces. J. Med. Chem., 2006, 49, 2713-2724.

[20] Kubinyi, H. QSAR: Hansch analysis and related approaches, VCH Publishers: Weinheim, New York, Basel, Cambridge, Tokyo, 1993; Vol. 1.

[21] Mitchell, T. Machine Learning; McGraw-Hill Science/Engineering/Math, 1997.

[22] Gaulton, A.; Bellis, L.J.; Bento, A.P.; Chambers, J.; Davies, M.; Hersey, A.; Light, Y.; McGlinchey, S.; Michalovich, D.; Al-Lazikani, B.; Overington, J.P. ChEMBL: a large-scale bioactivity database for drug discovery. Nucleic Acids Res., 2012, 40(DI), D1100-1107. 
[23] Karelson, M. Molecular Descriptors in QSAR/QSPR; Wiley-Interscience: New York, 2000.

[24] Riera-Fernandez, I.; Martin-Romalde, R.; Prado-Prado, F.J.; Escobar, M.; Munteanu, C.R.; Concu, R.; Duardo-Sanchez, A.; Gonzalez-Diaz, H. From QSAR models of drugs to complex networks: state-of-art review and introduction of new markov-spectral moments indices. Curr. Top. Med. Chem., 2012, 8, 927-960.

[25] Munteanu, C.R.; Fernandez-Blanco, E.; Seoane, J.A.; Izquierdo-Novo, P.; RodriguezFernandez, J.A.; Prieto-Gonzalez, J.M.; Rabunal, J.R.; Pazos, A. Drug discovery and design for complex diseases through QSAR computational methods. Curr. Pharm. Des., 2010, 16(24), $2640-2655$.

[26] Garcia, I.; Munteanu, C.R.; Fall, Y.; Gomez, G.; Uriarte, E.; Gonzalez-Diaz, H. QSAR and complex network study of the chiral HMGR inhibitor structural diversity. Bioorg. Med. Chem., 2009, 17(1), 165-175.

[27] Prado-Prado, F.J.; Martinez de la Vega, O.; Uriarte, E.; Ubeira, F.M.; Chou, K.C.; GonzalezDiaz, H. Unified QSAR approach to antimicrobials. 4. Multi-target QSAR modeling and comparative multi-distance study of the giant components of antiviral drug-drug complex networks. Bioorg. Med. Chem., 2009, 17(2), 569-575.

[28] Prado-Prado, F.J.; Garcia, I.; Garcia-Mera, X.; Gonzalez-Diaz, H. Entropy multi-target QSAR model for prediction of antiviral drug complex networks. Chemom. Intell. Lab. Syst., 2011, 107(2), 227-233.

[29] Prado-Prado, F.J.; Borges, F.; Uriarte, E.; Perez-Montoto, L.G.; Gonzalez-Diaz, H. Multi-target spectral moment: QSAR for antiviral drugs vs. different viral species. Anal. Chim. Acta, 2009, 651(2), 159-164.

[30] Tenorio-Borroto, E.; Penuelas Rivas, C.G.; Vasquez Chagoyan, J.C.; Castanedo, N.; PradoPrado, F.J.; Garcia-Mera, X.; Gonzalez Diaz, H. ANN multiplexing model of drugs effect on macrophages; theoretical and flow cytometry study on the cytotoxicity of the antimicrobial drug G1 in spleen. Bioorg. Med. Chem., 2012, 20, 6181-6194.

[31] Prado-Prado, F.J.; Ubeira, F.M.; Borges, F.; Gonzalez-Diaz, H. Unified QSAR \& networkbased computational chemistry approach to antimicrobials. ii. multiple distance and triadic census analysis of antiparasitic drugs complex networks. J. Comput. Chem., 2010, 31(1), 164173.

[32] Prado-Prado, F.J.; Gonzalez-Diaz, H.; Santana, L.; Uriarte, E. Unified QSAR approach to antimicrobials. Part 2: predicting activity against more than 90 different species in order to halt antibacterial resistance. Bioorg. Med. Chem., 2007, 15(2), 897-902.

[33] Prado-Prado, F. J.; Gonzalez-Diaz, H.; Martinez de la Vega, O.; Ubeira, F.M.; Chou, K.C. Unified QSAR approach to antimicrobials. Part 3: First multi-tasking QSAR model for InputCoded prediction, structural back-projection, and complex networks clustering of antiprotozoal compounds. Bioorg. Med. Chem., 2008, 16, 5871-5880.

[34] Gonzalez-Diaz, H.; Prado-Prado, F.; Ubeira, F.M. Predicting antimicrobial drugs and targets with the MARCH INSIDE approach. Curr. Top. Med. Chem., 2008, 8(18), 1676-1690.

[35] Gonzalez-Diaz, H.; Prado-Prado, F. Unified QSAR and network based computational chemistry approach to antimicrobials, part 1: multispecies activity models for antifungals. J. Comput. Chem., 2008, 29, 656-657.

[36] Fernandez-Lozano, C.; Cuinas, R.F.; Seoane, J.A.; Fernandez-Blanco, E.; Dorado, J.; Munteanu, C.R. Classification of signalling proteins based on molecular star graph descriptors using Machine Learning models. J. Theor. Biol., 2015, 384, 50-58.

[37] Vazquez, J.M.; Aguiar, V.; Seoane, J.A.; Freire, A.; Serantes, J.A.; Dorado, J.; Pazos, A.; Munteanu, C.R. Star graphs of protein sequences and proteome mass spectra in cancer prediction. Curr. Proteomics, 2009, 6(4), 275-288.

[38] Munteanu, C.R.; Magalhaes, A.L.; Uriarte, E.; Gonzalez-Diaz, H. Multi-target QPDR classification model for human breast and colon cancer-related proteins using star graph topological indices. J. Theor. Biol., 2009, 257(2), 303-311.

[39] Munteanu, C.R.; Gonzalez-Diaz, H.; Magalhaes, A.L. Enzymes/non-enzymes classification model complexity based on composition, sequence, 3D and topological indices. J. Theor. Biol., 2008, 254(2), 476-482.

[40] Fernandez-Lozano, C.; Gestal, M.; Pedreira-Souto, N.; Postelnicu, L.; Dorado, J.; Munteanu, C.R. Kernel-based feature selection techniques for transport proteins based on star graph topological indices. Curr. Top. Med. Chem., 2013, 13(14), 1681-1691.

[41] Fernandez-Lozano, C.; Gestal, M.; Gonzalez-Diaz, H.; Dorado, J.; Pazos, A.; Munteanu, C.R. Markov mean properties for cell deathrelated protein classification. J. Theor. Biol., 2014, 349, 12-21. 
[42] Marrero-Ponce, Y.; Ortega-Broche, S.E.; Echeverria Diaz, Y.; Alvarado, Y.J.; Cubillan, N.; Casas Cardoso, G.; Torrens, F.; Perez-Gimenez, F. Nucleotide's bilinear indices: Novel biomacromolecular descriptors for bioinformatics studies of nucleic acids. I. Prediction of paromomycin's affinity constant with HIV-1 Psi-RNA packaging region. J. Theor. Biol., 2009, 259(2), 229-241

[43] Marrero-Ponce, Y.; Nodarse, D.; Gonzalez-Diaz, H.; Ramos de Armas, R.; Romero-Zaldivar, V.; Torrens, F.; Castro, E.A. Nucleic acid quadratic indices of the "macromolecular graph's nucleotides adjacency matrix". Modeling of footprints after the interaction of paromomycin with the HIV-1 $\Psi$-RNA packaging region. Int. J. Mol. Sci., 2004, 5, 276-293.

[44] Deng, Y.L.; Han, X.F.; Tang, S.X.; Xiao, W.J.; Tan, Z.L.; Zhou, C.S.; Wang, M.; Kang, J.H. Magnolol and honokiol regulate the calcium-activated potassium channels signaling pathway in Enterotoxigenic Escherichia coli-induced diarrhea mice. Eur. J. Pharmacol., 2015, 755, 66-73.

[45] Yang, J.; Dong, S.; Zhu, H.; Jiang, Q.; Yang, J. Molecular and expression analysis of manganese superoxide dismutase (Mn-SOD) gene under temperature and starvation stress in rotifer Brachionus calyciflorus. Mol. Biol. Rep., 2013, 40(4), 2927-2937.

[46] Smith, T.C.; Frank, E. Introducing machine learning concepts with WEKA. In: Statistical Genomics: Methods and Protocols; Springer: New York, NY, 2016; pp 353-378.

[47] Cao, D.S.; Xiao, N.; Xu, Q S.; Chen, A.F. Rcpi: R/Bioconductor package to generate various descriptors of proteins, compounds and their interactions. Bioinformatics, 2015, 31(2), 279-281.

[48] Team, R.D.C. $R$ : A language and environment for statistical computing. $R$ Foundation for Statistical Computing, Vienna, Austria, R Foundation for Statistical Computing: Vienna, Austria, 2016.

[49] Fan, R.-E.; Chang, K.-W.; Hsieh, C.-J.; Wang, X.-R.; Lin, C.-J. LIBLINEAR: A library for large linear classification. J. Mach. Learn. Res., 2008, 9, 1871-1874.

[50] Langley, P.; Iba, W.; Thompson, K. In: An analysis of Bayesian classifiers, Proceedings of the Tenth National Conference on Articial Intelligence; San Jose, CA, AAAI: San Jose, CA, 1992; pp 223-228.

[51] Kohavi, R. The Power of Decision Tables. In: Proceedings of $8^{\text {th }}$ European Conference on Machine Learning; Springer-Verlag: Heraclion, Greece, 1995; pp 174-189.

[52] Breiman, L. Random forests. Machine Learning, 2001, 45(1), 5-32.

[53] Witten, I.; Frank, E. Data Mining: Practical Machine Learning Tools and Techniques, Second Edition (Morgan Kaufmann Series in Data Management Systems), Morgan Kaufmann, 2005.

[54] Biau, G. Analysis of a random forests model. J. Machine Learn. Res., 2012, 13, 1063-1095.

[55] Jin, H. Using AUC and Accuracy in Evaluating Learning Algorithms. In: IEEE Transactions on Knowledge and Data Engineering, 2005, 17(3), 299-310.

[56] Zhou, H.Y.; Shin, E.M.; Guo, L.Y.; Youn, U. J.; Bae, K.; Kang, S.S.; Zou, L.B.; Kim, Y.S. Anti-inflammatory activity of 4-methoxyhonokiol is a function of the inhibition of iNOS and COX-2 expression in RAW 264.7 macrophages via NF-kappa B, JNK and p38 MAPK inactivation. Eur. J. Pharmacol., 2008, 586(1-3), 340-349.

[57] Yang, T.C.; Zhang, S.W.; Sun, L.N.; Wang, H.; Ren, A.M. Magnolol attenuates sepsis-induced gastrointestinal dysmotility in rats by modulating inflammatory mediators. World $J$. Gastroenterol., 2008, 14(48), 7353-7360.

[58] Urso, M.L.; Clarkson, P.M. Oxidative stress, exercise, and antioxidant supplementation. Toxicology, 2003, 189(1-2), 41-54.

[59] Giustarini, D.; Dalle-Donne, I.; Tsikas, D.; Rossi, R. Oxidative stress and human diseases: Origin, link, measurement, mechanisms, and biomarkers. Crit. Rev. Clin. Lab. Sci., 2009, 46(56), 241-281.

[60] Hajiani, M.; Golestani, A.; Shariftabrizi, A.; Rastegar, R.; Payabvash, S.; Salmasi, A.H.; Dehpour, A.R.; Pasalar, P. Dose-dependent modulation of systemic lipid peroxidation and activity of antioxidant enzymes by vitamin E in the rat. Redox Rep., 2008, 13(2), 60-66.

[61] Lambert, J.D.; Elias, R.J. The antioxidant and pro-oxidant activities of green tea polyphenols: A role in cancer prevention. Arch. Biochem. Biophys., 2010, 501(1), 65-72.

[62] Molina, M.F.; Sanchez-Reus, I.; Iglesias, I.; Benedi, J. Quercetin, a flavonoid antioxidant, prevents and protects against ethanolinduced oxidative stress in mouse liver. Biol. Pharm. Bull., 2003, 26(10), 1398-1402.

[63] Lubos, E.; Loscalzo, J.; Handy, D.E. Glutathione peroxidase-1 in health and disease: from molecular mechanisms to therapeutic opportunities. Antioxid. Redox Signaling, 2011, 15(7), 1957-1997.

[64] Pedraza-Chaverri, J.; Granados-Silvestre, M.D.; Medina-Campos, O.N.; Maldonado, P.D.; Olivares-Corichi, I.M.; Ibarra-Rubio, M.E. Post-transcriptional control of catalase expression in garlic-treated rats. Mol. Cell. Biochem., 2001, 216(1-2), 9-19. 
[65] Yang, J.; Dong, S.; Jiang, Q.; Kuang, T.; Huang, W.; Yang, J. Changes in expression of manganese superoxide dismutase, copper and zinc superoxide dismutase and catalase in Brachionus calyciflorus during the aging process. PLoS ONE, 2013, 8(2), e57186. 